\title{
Resonance scattering in waveguides: Acoustic scatterers
}

\author{
Philip M. Carrion and Marco Antonio P. de Brito \\ UFBA/IGEO/PPPG, Campus Universitário, Federação-40210, Salvador-BA, Brazil
}

(Received 31 January 1989; accepted for publication 29 September 1989)

\begin{abstract}
In this paper, acoustic scattering in shallow and deep inhomogeneous waveguides is analyzed. The full acoustic wave equation that describes the scattered and reflected wave fields, as well as all multipathing within the scatterer and the waveguide, is employed. The explicit finite difference time integration and the $k-w$ transform in the space domain (pseudospectral method) were utilized. Properly chosen boundary conditions enabled the authors to model both shallow and deep oceans. The pseudospectral method was compared with the explicit finite difference technique (see Appendix). The pseudospectral method can be valuable for modeling different underwater wave phenomena: It is characterized by much smaller numerical dispersion than the conventional finite difference method. The results show that in shallow ocean, strong resonance coupling between the underwater scatterer and the waveguide may occur. An important conclusion of this paper is that in a limited aperture experiment when the acoustic reflections are beyond the recording aperture (due to the finite length of the recording cable), the measured data are mainly represented by the primary diffraction arrivals and "diffraction resonances." In this paper, a detailed discussion will be given on the acoustic scattering in an inhomogeneous waveguide. (For the sake of simplicity, an acoustic scatterer of the rectangular shape was considered.) Different complexities (elastic scatterers, more complicated structures of the index of refraction in the water, etc.) will be compounded in the original model and reported elsewhere.
\end{abstract}

PACS numbers: 43.30.Gv, 43.30.Hw, 43.30.Bp, 43.20.Mv

\section{INTRODUCTION}

Propagation of sound in the ocean can be treated as a waveguide problem. The sea surface can be considered with a great deal of accuracy as a pressure-release surface, so all acoustic energy generated below the sea surface is reflected back with opposite polarity (for pressure). These reflections are called ghosts. The sea surface, due to its roughness, may generate significant noise that is imposed on the returned signals. The propagation speed in the water can be rather complex: this leads to additional scattering and diffraction on large- and small-scale inhomogeneities (large-scale inhomogeneities are much greater than the predominant wavelength, whereas small-scale inhomogeneities are of the order or less than the characteristic wavelength). Recall that small-scale scattering is related to the so-called apparent attenuation. As the result of apparent attenuation, a pulse propagating through a loss-free medium with a highly discontinuous sound-speed profile is getting broader in width and smaller in amplitude.

The recorded underwater signals are typically nonstationary; their stochastic properties are functions of time. The effects of nonstationarity of the returned signals can be wellestimated in the $t-w$ domain. $^{\prime}$

When the generated sound reaches the sea bottom, it is reflected or scattered from marine sediments. The backpropagating signal goes back to the sea surface and is totally reflected by the sea surface regardless of the angle of incidence. This signal will be bouncing between the sea surface and the sea bottom causing waveguide resonances. The resonance characteristics depend upon the angle of propagation, the reflection coefficient at the marine sediments, and the refraction index in the ocean.

Carrion and Satkowiak, ${ }^{2}$ using the cepstrum analysis, showed recently that in the range of $20-80 \mathrm{kHz}$, marine sediments behave as a stack of continuous scatterers rather than discrete reflectors. This means that there are no distinct interfaces in the subbottom medium that reflect the generated energy, rather the incident energy is scattered by continuously distributed scatterers in the marine sedimentary environment. Carrion and Wilbur, ' using the coherency measure between the sea-surface and the sea-bottom reflections, found that for mid-and small-grazing angles, the sea bottom behaves like the sea surface that reflects virtually all energy regardless of the frequency content of the initially generated signal. For larger grazing angles, the coherence between these signals fails for the range of high frequencies, which are attenuated in marine sediments. This means that the waveguide resonances will be especially significant if the incident angle is near or exceeds the critical angle. For angles below the critical angle, the amplitudes of waveguide bouncing waves decrease due to transmission losses in the subbottom environment (quasiresonances).

Theoretical aspects of acoustic and elastic resonance scattering was studied by different authors. Important contributions to this subject include Flax et al. ${ }^{3,4}$ Gaunaurd, ${ }^{5}$ Frisk and Uberall, ${ }^{6}$ and Werby and Green ${ }^{7}$ among others.

Hackman and Sammelmann ${ }^{8}$ have recently presented a rigorous study of acoustic scattering in range-independent waveguides based on the $T$-matrix formalism. Their model included an elastic spherical shell in inhomogeneous layered 
waveguides whose square of the index of refraction was linear with respect to depth. In the previous work, Sammelmann and Hackman ${ }^{9}$ computed scattering responses from the elastic shell in a homogeneous waveguide bounded by the pressure-release sea surface and the rigid sea bottom. (The rigid sea bottom was chosen to avoid the branch cuts of Green's functions. )

In a typical sonar experiment, a horizontal array of hydrophones is towed behind a ship and used as sonar receivers. ${ }^{10}$ Thus it is necessary to analyze resonance scattering in waveguides detected by a towing array of sonar receivers with limited recording aperture.

In this paper, we study scattering in waveguides using a string of hydrophones both in shallow and deep ocean. The numerical algorithm used in the paper is neither limited by the choice of frequencies nor by the geometry of the model. In fact, this study can be extended without much difficulty to range-dependent waveguide resonances and more complicated geometry of the underwater object.

The method described in the paper can be an alternative to the $T$-matrix formalism since it is rather flexible with regards to the choice of the boundary conditions for deep and shallow ocean.

Usually, the towing arrays are characterized by a limited aperture so a significant portion of the reflections from the target might be missing. In this paper, we studied effects of the limited aperture and introduced the concept of the diffraction resonances that constitute a significant contribution to the recorded data.

An important conclusion of this paper is that in shallow ocean, strong resonance coupling between the waveguide and the scatterer may occur. Our results show that the resonance coupling in deep ocean is small and typically can be ignored.

Although the examples presented in the paper are limited by acoustic scattering, different complexities will be included in the model to make it more realistic.

In the Appendix, we will compare the Fourier method with the conventional finite difference modeling. It will be shown that the pseudospectral method can be very valuable for modeling different underwater wave phenomena. For example, for the same frequencies, numerical dispersion in the pseudospectral method does not depend on the angle of propagation and is usually much smaller than in the conventional finite difference techniques.

\section{SCATTERING THEORY}

Usually acoustic/elastic scattering is analyzed using the Green's-Betty theorem, where the scattering response is found by integration of Green's function, pressure, and their normal derivatives along the surface of the scatterer. The $T$ matrix formalism can then be employed. ${ }^{4}$ The $T$-matrix approach can be efficiently used for objects of different shapes whose basis functions are not complicated. For targets of arbitrary shapes, basis functions are no longer simple and this can lead to cumbersome calculations. Another approach to acoustic/elastic scattering is based on numerical solutions of the acoustic/elastic wave equations with appropriate initial (IC) and boundary (BC) conditions.
In this paper, we will consider the complete (full waveforms) acoustic wave equation that governs the propagation of pressure in liquids.

Wave propagation in a 2-D acoustic medium can be written as follows:

$$
\frac{\partial}{\partial x}\left(\frac{1 \partial P}{\rho \partial x}\right)+\frac{\partial}{\partial z}\left(\frac{1 \partial P}{\rho \partial z}\right)=\frac{1}{\rho c^{2}} \frac{\partial^{2} P}{\partial t^{2}}+S(t) \delta(x) \delta(z),
$$

where $P(x, z, t)$ is the pressure field, $c(x, z)$ is the propagation speed, $\rho(x, z)$ is the density, $S(t)$ is the source time function (sonar generted pulse), and $\delta^{2}(\cdot)$ describes the location of the sonar.

We use the explicit discretization in time (explicit-time scheme). The second-order time derivative can be presented as

$$
\frac{\partial^{2} P_{n, m}^{l}}{\partial t^{2}}=\frac{P_{n, m}^{l+1}-2 P_{n, m}^{l}+P_{n, m}^{l-1}}{\Delta t^{2}}+O\left(\Delta t^{2}\right),
$$

where $n$ and $m$ represent sampling in the $x$ and $z$ directions and $l$ is the sampling rate in time. The time step was conformed to the LCF stability condition. ${ }^{11}$

We use the FFT method previously discussed by Kosloff and Baysal. ${ }^{12}$ This method requires fewer number of grid points to achieve the same accuracy than other techniques and consists of the following steps:

(a) apply the FFT to the wave field (e.g., pressure);

(b) multiply the results by $i k_{x}$ and $i k_{z}$;

(c) apply the $\mathrm{FFT}^{-1}$, giving $\partial P / \partial x$ and $\partial P / \partial z$;

(d) multiply $\partial P / \partial x$ and $\partial P / \partial z$ by $1 / \rho$;

(e) apply the FFT to the products and multiply them by $i k_{x}$ and $i k_{z}$;

(f) apply the FFT ${ }^{-1}$ and obtain $(\partial / \partial x)(1 / \rho)(\partial P / \partial x)$ and $(\partial / \partial z)(1 / \rho)(\partial P / \partial z)$.

Remark: Due to the finite discretization of the model space, finite difference or finite element numerical schemes are characterized by the apparent numerical dispersion that is expressed by the difference in the phase and group velocities. " For pseudospectral methods, this dispersion can be negligibly small. Initial conditions evolve the causality principle, whereas the boundary conditions at the sea bottom for the deep ocean are chosen to be absorbing and for shallow ocean, express the continuity of pressure across the boundary.

Usually, acoustic scattering is considered in the WKBJ approximation. In the high-frequency approximation, the large portion of data can be described in terms of the WKBJ (geometrical optics) approximation using the phase and the amplitude functions. The phase function satisfies the eikonal equation, whereas the amplitude function is governed by the transport equation. For the refraction index of different complexity, solutions to the transport equation are typically singular due to occurrence of caustics in the medium of consideration. In this study, we do not operate in the WKBJ approximation and thus we do not expect that caustics do occur.

We will consider limited aperture towing arrays that do not detect the reflections from the scatterer that are beyond the recorded aperture. We advocate an interesting result, that in the case of limited aperture array, the recorded data 
mainly contain diffraction and diffraction resonance arrivals.

In the next section, we will give the description of the acoustic numerical experiment in deep ocean where the resonance coupling is small.

\section{RESONANCE SCATTERING IN INHOMOGENEOUS DEEP OCEAN}

In this section, we will consider deep ocean. In order to better understand resonance scattering, the model was chosen in such a way that the reflections from the boundaries of the underwater object placed in the waveguide were missing due to the limited recording aperture. The sea surface is presented by the free surface (pressure-release surface). At the free-surface pressure vanishes at all points.

In order to eliminate the influence of the sea bottom, absorbing boundary conditions were incorporated.

Absorbing boundary conditions were set up in the coat layer. The loss-free acoustic wave equation in this "layer" was substituted by the viscoacoustic equation with a damping parameter. When this parameter is large, and the coat layer is small, we may anticipate some reflections from the "absorbing" boundaries. The best results turn out if the compromise between the value of the damping parameter and the size of the coat layer is achieved. Properly chosen damping parameter and the thickness of the coat layer enable one to suppress unwanted reflections from the sea bottom and thus to accurately analyze the deep-ocean responses. This can be done using the trial and error method. In our case, 30 grid points were assigned to the coat layer. The pressure field $\boldsymbol{P}_{c}$ in the coat layer is presented by

$$
P_{c} \approx \exp [-\alpha(N-i)],
$$

where $N$ is the total number of the grid points in the coat layer and $\alpha$ is a damping parameter.

Let us introduce the dimensionless quantity $K a$ (where $a$ is the size of the scatterer). For our model, $K a=4.86$. Along with $K a$, we will consider another parameter $K L(L$ is the range for the sonar experiment). In our case, $K L$ is 61.83 , so $K L \gg K a$ (this defines the farfield region).

Figure 1 is the model where the source is located at depth $90 \mathrm{~m}$ below the sea surface. The scatterer that has the shape of a square is placed inside the sound layer at a depth of $330 \mathrm{~m}$ below the sea surface. The side of the scatterer is $30 \mathrm{~m}$. The width of the sound layer is $190 \mathrm{~m}$. The receivers were placed at the same depth with the source. The nearest offset (the distance between the source and the first hydrophone) was $60 \mathrm{~m}$, so the length of the towing array was $150 \mathrm{~m}$. The source signature is presented in Fig. 2 that shows the zerophase Ricker-type wavelet. The high cutoff frequency was $245 \mathrm{~Hz}$. Figure 3 shows the discretization of the model scatterer. The target has 10 grid points per side.

Figure 4 shows the numerical result of scattering in deep ocean recorded by 50 hydrophones spaced $3 \mathrm{~m}$ apart. The first arrival (marked by "1") is the direct wave traveling from the source to the receivers. The second arrival is the ghost (reflection from the sea surface), the third arrival is the reflection from the top of the sound layer. The fourth arrival is the second-order (first-order multiple) reflection

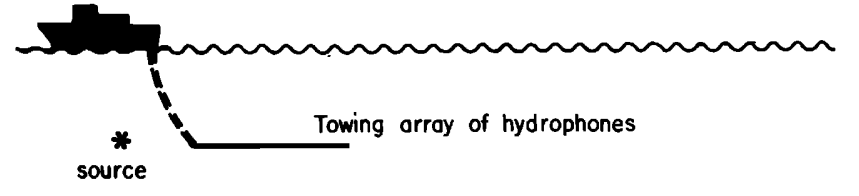

$V=1482.25$

$p=1.0$

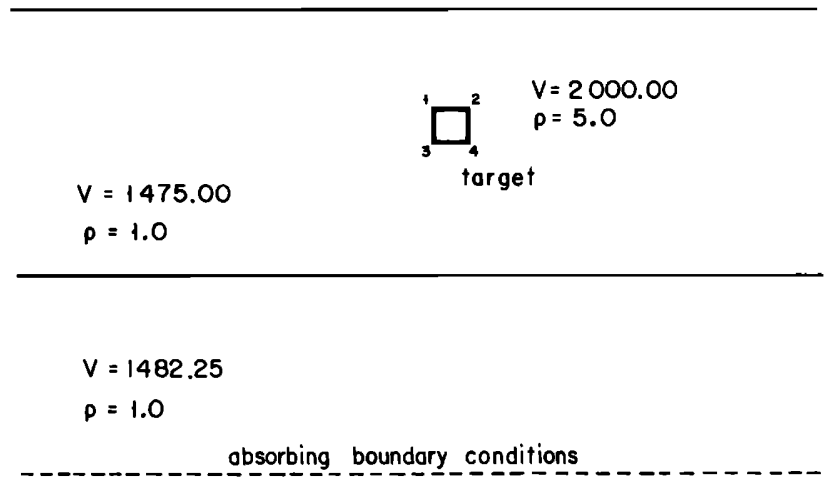

FIG. 1. Model of the sonar experiment in deep ocean. Towing array of hydrophones is placed above the target. Absorbing boundary conditions are imposed on the sea bottom to suppress reflections from the sea fioor.

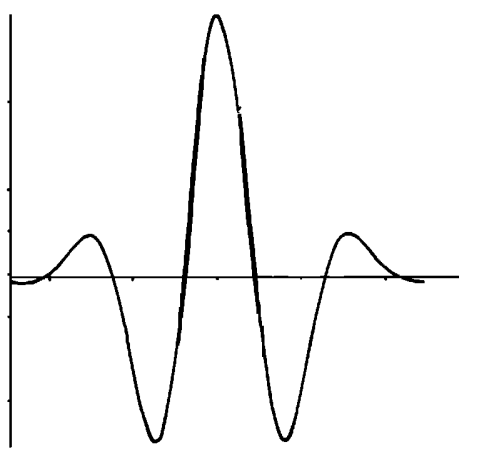

FIG. 2. Zero-phase Ricker wavelet used in the numerical modeling.

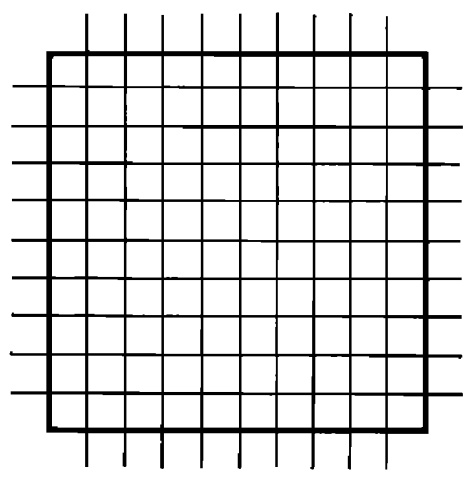

FIG. 3. Discretization in the model space. The size of the cell was $3 \mathrm{~m}$ : 10 grid points per side of the scatterers. 


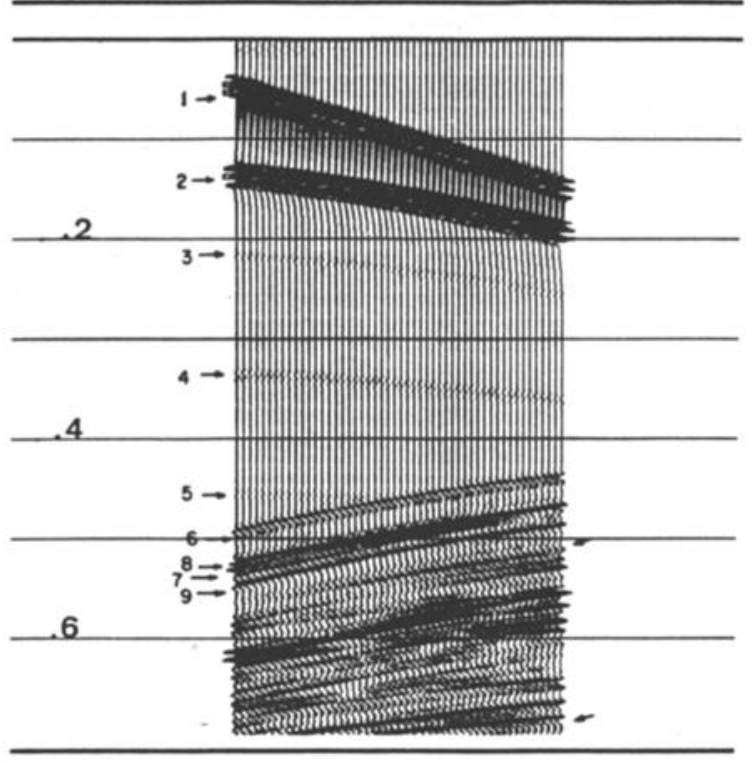

FIG. 4. Response of the towing array (at the 50th hydrophone). Arrows indicate the resonance scattering. Letter $d$ indicates diffraction arrivals from the corners.

from the top of the sound layer. Number "5" marks the reflection from the bottom of the sound layer. Number " 6 " stands for the diffraction curve from the first corner of the scatterer and the seventh arrival is the diffraction arrival coming from the second corner of the scatterer (see Fig. 1). Number " 8 " depicts the diffraction event from the third corner and finally number "9" is the diffraction event from the fourth corner. It is important to note that the scatterer shows strong resonance pattern (events between arrows in this figure). Figure 5(a) depicts the first hydrophone reading. It shows that all events below the $0.55 \mathrm{~s}$ correspond to internal resonances within the scatterer. Figure 5(b) is the response recorded by the last hydrophone located at $210 \mathrm{~m}$ away from the source. Events below $0.5 \mathrm{~s}$ are the resonances within the scatterer. Our results computed for the deep-ocean model indicate that the resonance coupling between the scatterer and the waveguide is negligibly small.

\section{DIFFRACTION RESONANCES}

When the generated waves impinge on the boundaries of the scatterer, the scatterer excites the so-called Huygens' secondary sources. The transmitted energy can be decomposed into the vertically and laterally propagating pressure fields: This means that internal resonances can be considered as a combination of horizontal and vertical resonating waves. In accordance with the chosen geometry presented in Figs. 1 and 3 , one can realize that due to the limited aperture of the recording cable, internal vertical and horizontal resonances will be not recorded by the towing array of hydrophones. This means that only nondirectional perturbations will be recorded. The only areas that generate nondirectional energy are corners of the scatterer. Thus internal resonances within the scatterer will be detected due to point diffractors (corners). These events we call the diffraction resonances that are well seen in Fig. 4 where the geometry of the diffrac-
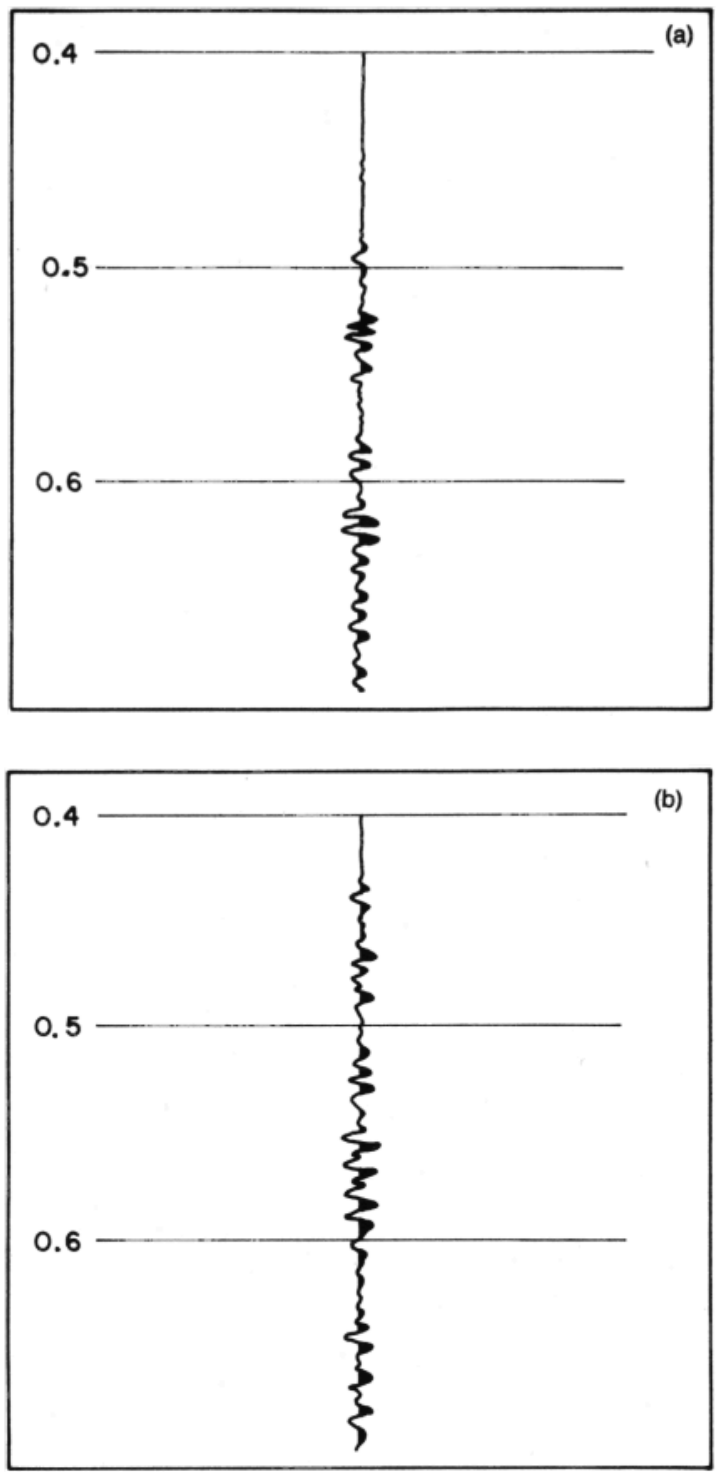

FIG. 5. (a) Recorded signal at the first hydrophone shows rather complicated resonance signature (marked between arrows). (b) Recorded signal at the 50th (the furthest) hydrophone shows the resonant signature of bounced waves.

tion response coincides exactly with the geometry of the diffraction resonances marked by two arrows. This suggests that the resonance pattern recorded by the towing array comes from the resonating corners of the target. Figure 6 demonstrates this concept. One can observe that recorded resonances have large amplitudes and a steady pattern in time. These are characteristics of standing waves. The response from underwater objects in a limited aperture experiment with missing reflections is therefore mainly characterized by the diffraction arrivals and the internal resonance pattern.

\section{SHALLOW OCEAN SCATTERING IN INHOMOGENEOUS WAVEGUIDES}

\section{A. Resonance coupling}

Remark: By definition, resonances are determined by bouncing waves between two boundaries. A perfect example 


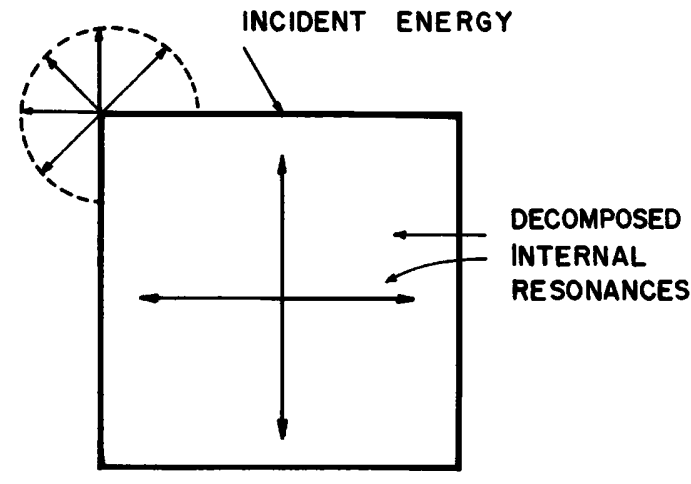

FIG. 6. Internal resonances are not detected due to the limited aperture of the towing array. Responses from the resonating corners have the same travel time geometry as primary diffraction arrivals.

of the resonance is the wave traveling between the sea surface and the sea bottom. In fact, the number of resonance cycles depends on the reflection coefficients. If the reflection coefficient of the sea bottom is small, one deals with damping resonances. The resonance coupling is an event that is a sum of two resonances. Its signature contains periodic waveforms of different periods. In the frequency domain, resonances correspond to singular solutions for the amplitude function (regular poles) for frequencies in the neighborhood of the natural frequency of the waveguide. Resonances can be expressed in terms of the multiple generator operator. Let us consider the multiple generator that corresponds to normal incidence. Bouncing energy in the waveguide can be expressed by the following periodic function:

$$
M=\sum_{j=1}^{\infty}(-1)^{j} a_{j} \delta\left(t-T_{j}\right),
$$

where

$$
T_{j}=2 j \int_{0}^{a} \frac{d Z}{c(z)}
$$

is the two-way travel time and $a_{j}$ are the reflection coefficients. For example, $a_{1}=R$, where $R$ is the reflection coefficient from the sea bottom and $a_{2}=R^{2}$ and so on. So (4) can be rewritten as

$$
M=\sum_{j=1}^{\infty}(-1)^{j} R^{j} \delta\left(t-T_{j}\right)
$$

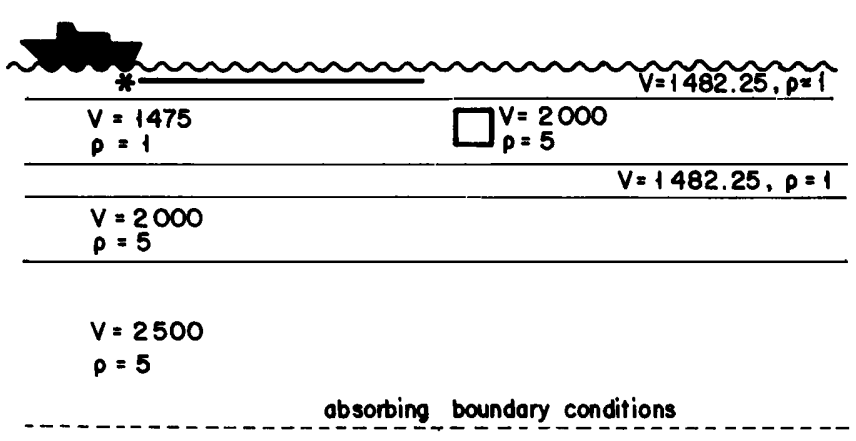

FIG. 7. Model of the shallow ocean with the target placed in the sound layer.
Suppose now that the multiple generator corresponding to the scatterer is

$$
M_{x}=\sum_{j=1}^{\infty}(-1)^{j} b_{j} \delta\left(t-\widetilde{T}_{j}\right),
$$

where $b_{j}$ is the reflection coefficient from the target boundaries and $\widetilde{T}_{j}$ is the period of internal resonances. If

$$
T_{j}=m \widetilde{T}_{j},
$$

where $m$ is an integer, the internal resonances and the resonances in the waveguide will then be tangled. Our results show that this coupling can be substantial, since the amplitudes of the internal resonances match the amplitudes of the waveguide resonances. In order to untangle these resonances, the waveguide ringing can be suppressed. ${ }^{13}$ For multichannel sonar experiments, the resonances can be decoupled using the differences in the geometries of the detected responses. The problem of decoupling needs separate treatment and thus is beyond the scope of the paper.

One of the most important problems in underwater acoustics is to deduce acoustic sources of energy and to determine the propagation characteristics of acoustic waves. For example, the sea bottom is transparent for acoustic waves. However, for near critical reflections, it resembles the sea surface. The sea-bottom response is not only a function of the angle but also depends on frequency: namely high frequencies can be absorbed in marine sediments at rather shallow depths so the reflection response will not contain the high-frequency component of its spectrum. As the first approximation, we will model the sea bottom as a two-layer acoustic medium.

Let us consider the following model. The scatterer of the square shape was placed in the sound layer whose width was $60 \mathrm{~m}$. The sonar was placed above the sound layer so the range was $300 \mathrm{~m}$. The depth of the ocean was $120 \mathrm{~m}$. The seabottom structure was modeled by two sediment layers with the sound speed being 2000 and $2500 \mathrm{~m} / \mathrm{s}$, respectively. The thickness of the first sediment layer was $45 \mathrm{~m}$. Figure 7 describes the model. Figure 8 is the reflection response record-

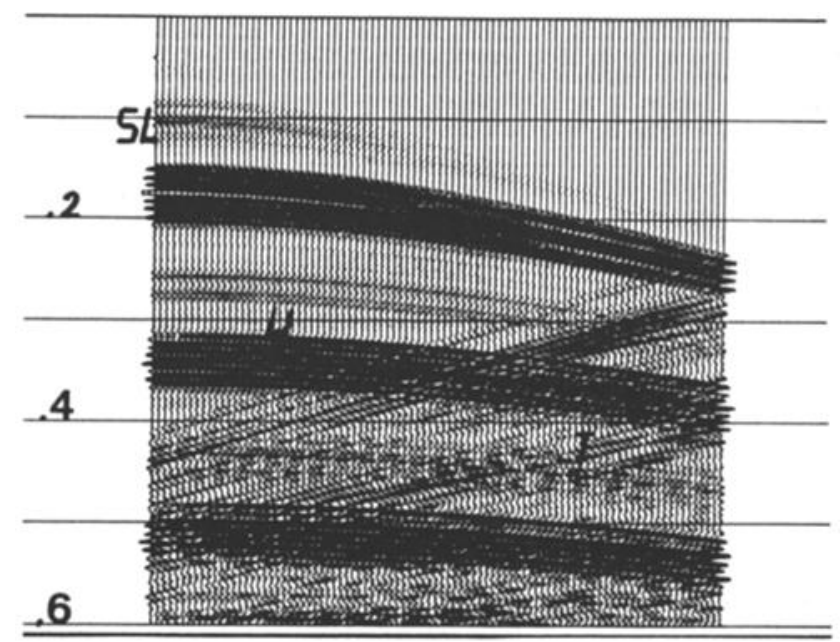

FIG. 8. Acoustic response from the shallow-ocean waveguide with the target. Letters SL mark the reflections from the sound layer. This figure shows strong coupling between the waveguide and the target resonances. 


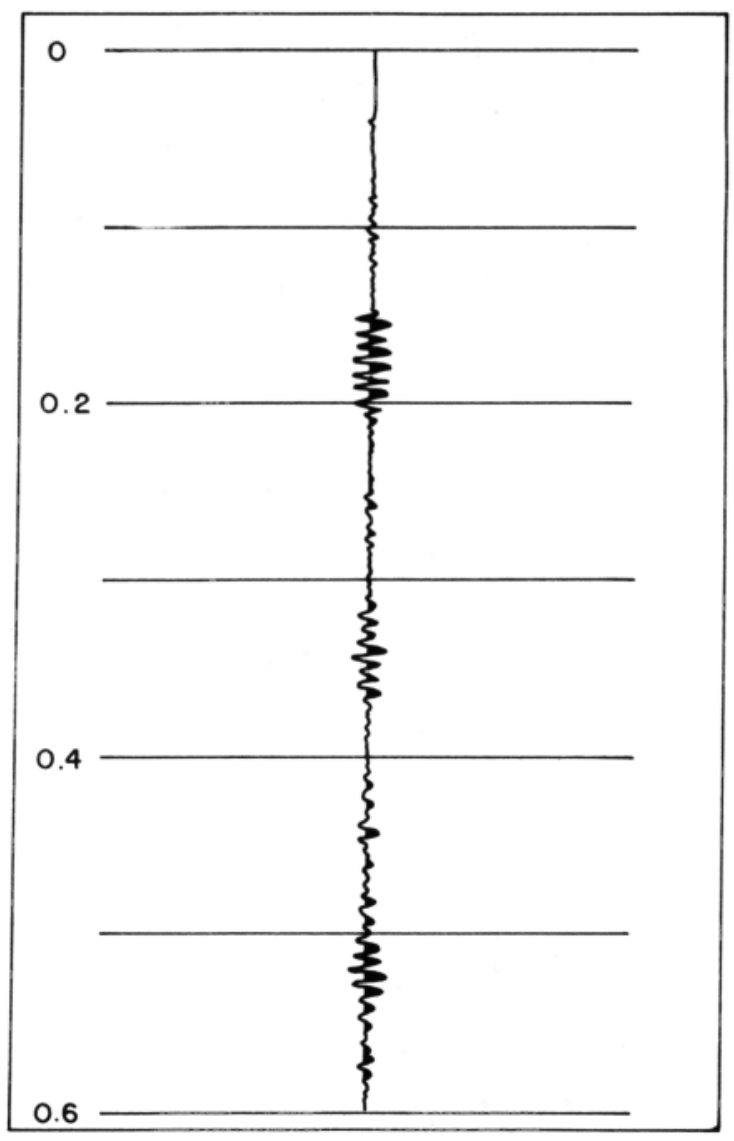

FIG. 9. Resonance coupling between the target and the waveguide for time greater than $0.4 \mathrm{~s}$.

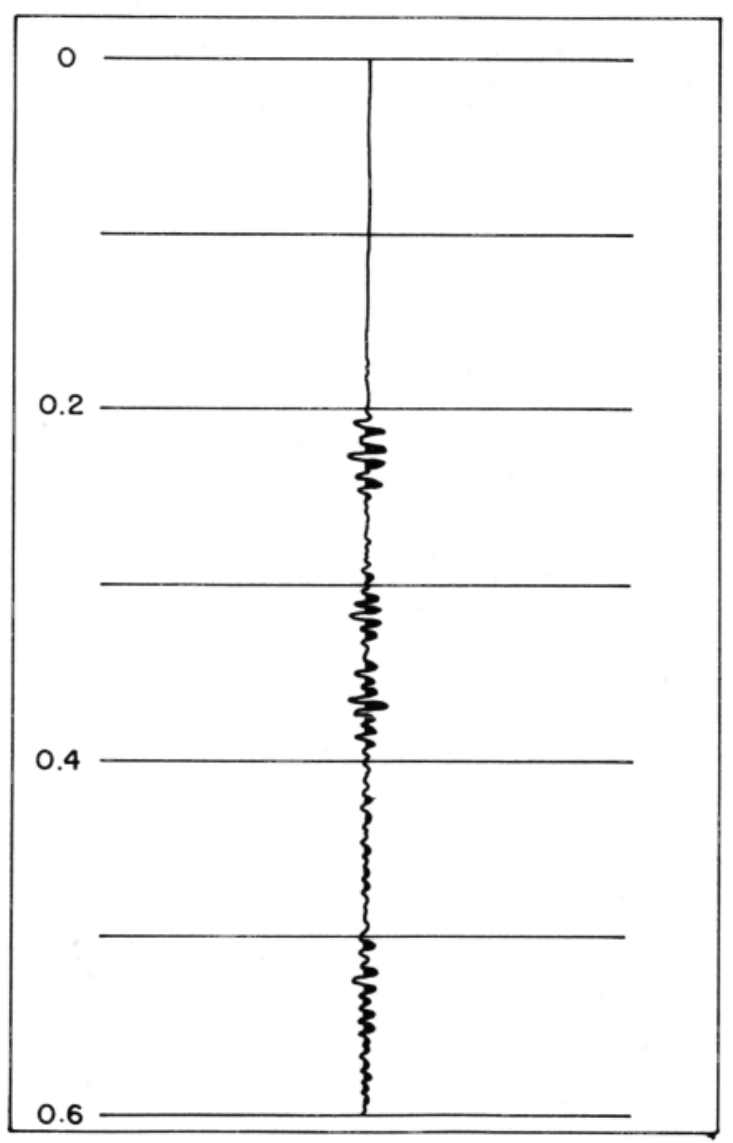

FIG. 10. Resonance coupling for time greater than $0.3 \mathrm{~s}$.

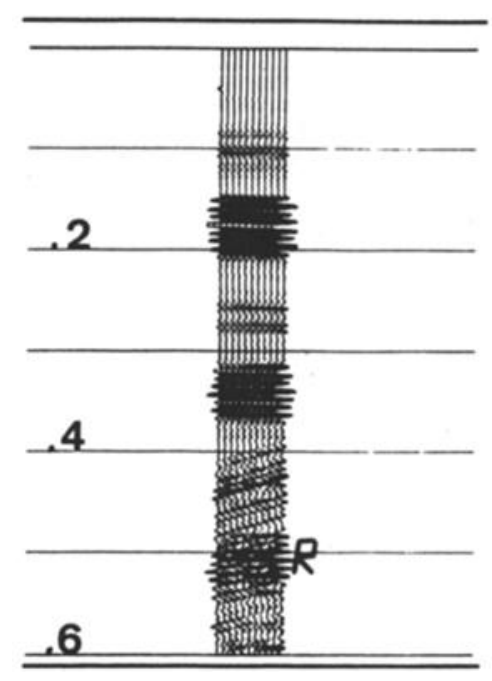

FIG. 11. Resonance coupling for the first 10 channels.

ed by the towing array of 90 hydrophones spaced $3 \mathrm{~m}$ apart. The direct wave was eliminated. Arrivals marked by letters SL correspond to the reflections from the sound layer. Since the sound layer is a low-velocity zone and thus does not have critical and supercritical reflections, the energy that corresponds to the critical reflections is missing. This figure shows strong resonance coupling between the waveguide and the internal scatterer resonances (waveguide resonances are marked by letter $W$ and the scatterer resonances are marked by letter $T$ ). Figure 9 is the recorded signal that shows the resonance coupling below $0.4 \mathrm{~s}$. Figure 10 is the resonance pattern for the channel No. 70 showing the resonance coupling for times later than $0.3 \mathrm{~s}$. Figure 11 demonstrates the resonance coupling marked by letter $R$ between the target and the waveguide for the first 10 channels, whereas Fig. 12 depicts the resonance coupling between the scatterer and the waveguide for the last 20 channels. One can see that this coupling is rather strong and obscures the scatterer resonances (compare with the deep-ocean results, Fig. 4). It is important to note that for a single channel, the waveguide

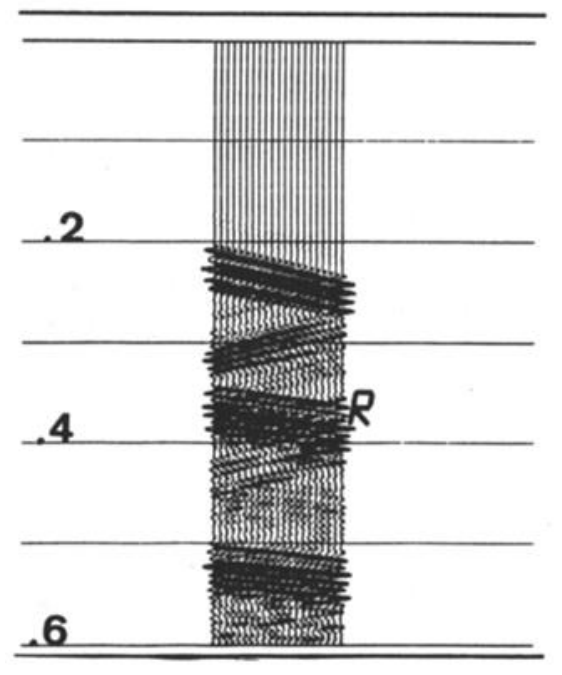

FIG. 12. Resonance coupling for the last 20 channels. 
and the scatterer resonances are difficult to separate. They can be better identified by a multichannel recording since the geometry (moveouts) of the scatterer resonances is typically different from the geometry of the waveguide resonances (see Fig. 12).

\section{v. CONCLUSION}

In this paper, we presented the results of the resonance scattering in inhomogeneous range-independent waveguides for both shallow and deep ocean. It was shown that acoustic scatterers can exhibit strong resonance patterns. We introduced a new concept of diffraction resonances that are caused by resonating point diffractors of the scatterer. The diffraction resonances occur despite the complexities of all multipathing within the target and have the same geometry in the $t-x$ domain as the primary diffractions.

In order to model deep ocean, we used absorbing boundary conditions at the sea bottom. The numerical method used in the paper is fast and allows one to increase the accuracy without increasing the number of grid points in the model space. Our results show that there is strong coupling between the internal scatterer resonances and the resonances in shallow waveguides.

In the Appendix, we compared the pseudospectral method and the finite difference technique. The main conclusion is that although the finite difference method can be, computationalwise, more economical, the numerical dispersion effects in the pseudospectral method are much less observed.

\section{ACKNOWLEDGMENTS}

We would like to thank PPPG/UFBA, CNPq (Brazilian National Science Foundation), Finep, Petrobras for support and encouragement. We thank Guenther Schwedersky for his participation at the initial state. PMC is grateful to Roger Hackman of the Naval Coastal Systems Center (Panama City, FL) for valuable discussions. We thank Dan Kosloff of the Tel-Aviv University for the use of his pseudospectral software subroutines that were helpful for modeling wave phenomena in waveguides. We thank the anonymous reviewer who suggested that we compare the pseudospectral and the finite difference techniques.

\section{APPENDIX: FINITE DIFFERENCE AND PSEUDOSPECTRAL METHODS}

In the previous section, we computed the resonance responses from the acoustic scatterer using the pseudospectral method. Let us compare this technique with the conventional finite difference method based on the explicit time-space integration. The finite difference technique has been well developed in underwater acoustics. ${ }^{14}$

Let us consider the stability and numerical dispersion of both schemes. The stability condition is usually expressed as

$$
\Delta t \leqslant \beta c / H \text {, }
$$

where $c$ is the minimum propagation speed, $H$ is the grid size, and $\Delta t$ the time step. Alford et al. ${ }^{15}$ showed that for finite difference schemes $\beta=\sqrt{3 / 8}$, while Kosloff and Baysal ${ }^{12}$ showed that $\beta=\sqrt{2} / \pi$ for the Fourier method. This means that a finer discretization is needed in the psuedospectral method to obtain the same stability.

Let us consider the numerical dispersion effects of the pseudospectral method. Figure A1 (a) shows the numerical dispersion for $\beta \approx 0.45$. The dotted curve shows the group velocity, whereas the solid line is the phase velocity. Figure Al (b) shows the numerical dispersion for $\beta=0.2$. One can see that in this case the numerical dispersion effects are almost missing. When we use the finite difference FD technique, the dispersion curve [Fig. A2(a)] for $\beta \approx 0.61$ shows significant dispersion for $k H>2$. It is interesting to see what happens when we use $\beta=0.2$. Figure A2(b) shows that the difference between the group and the phase velocity remains almost the same whereas the adverse effects of dispersion appear at lower frequencies. It is very interesting to underscore that in the FD method, the numerical dispersion is a function of angle of propagation while the pseudospectral method does not have this drawback.

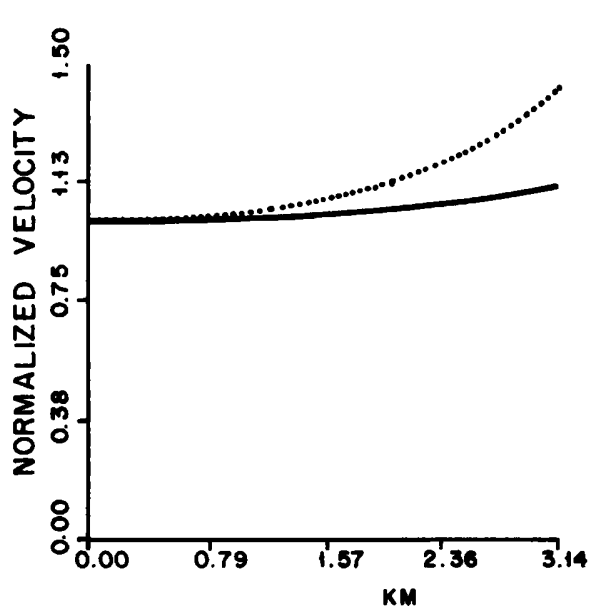

(a)

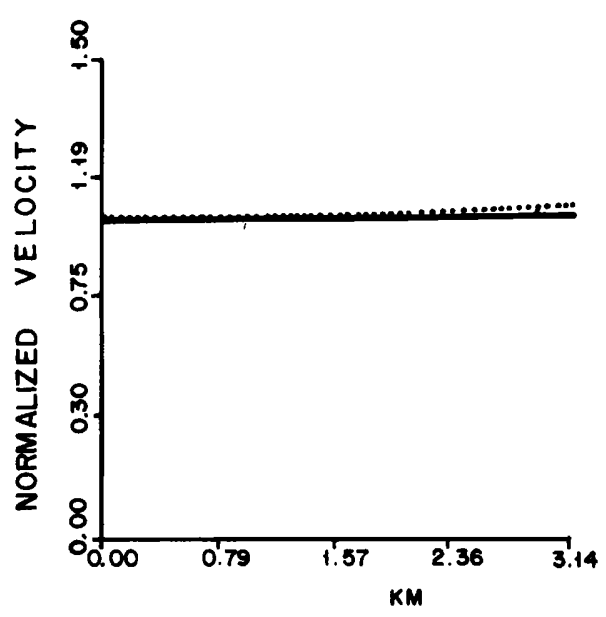

(b)
FIG. A1. (a) Dispersion curves in the pseudospectral method for $\beta=0.45$. Dotted line is group velocity. Solid line is the phase velocity plotted versus $K H$, where $H$ is the grid size. (b) Dispersion curves in the pseudospectral method for $\beta=0.2$. Dotted line is group velocity. Solid line is the phase velocity plotted versus $K H$. 


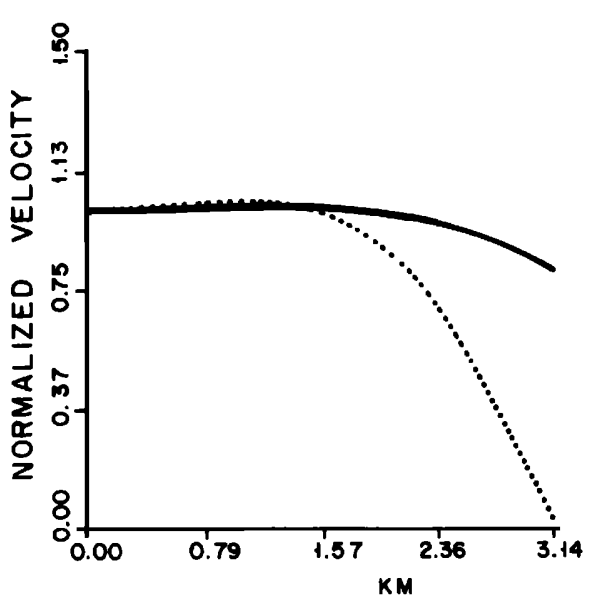

(a)

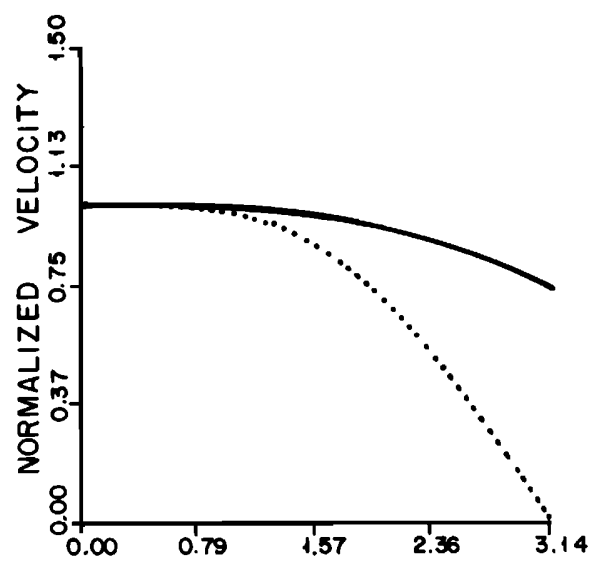

(b)
FIG. A2. (a) Dispersion curves in the finite difference method for $\beta=0.61$. Dotted line is group velocity. Solid line is the phase velocity plotted versus $K H$. (b) Dispersion curves in the finite difference method for $\beta=0.2$. Dotted line is group velocity. Solid line is the phase velocity plotted versus $K \boldsymbol{H}$.
'P. Carrion, and J. Wilbur, "Wide-aperture reflection acoustics: Sonar experiments in shallow water," J. Acoust. Soc. Am. 86, 1554-1559 (1989). ${ }^{2} \mathbf{P}$. Carrion, and L. J. Satkowiak, "Acoustic scattering from marine sediments in shallow water experiments," submitted to J. Acoust. Soc. Am.

${ }^{3}$ L. Flax, G. Gaunaurd, H. Uberall, "The theory of resonance scattering," in Physical Acoustics, edited by W. P. Mason and R. N. Thurston (Academic, New York, 1981), Vol. XV, Chap. 3, pp. 191-294.

${ }^{4}$ L. Flax, L. R. Dragonette, V. K. Varadan, and V. V. Varadan, “Analysis and computation of the acoustic scattering by an elastic prolate spheroid obtained from the T-matrix formulation," J. Acoust. Soc. Am. 71, 10771082 (1982).

${ }^{5}$ G. Gaunaurd, "Resonance theory of elastic and viscoelastic wave-scattering and its applications to the spherical cavity in absorptive media," in Modern Problems in Elastic Wave Propagation, edited by J. Miklowitz and J. Achenbach (Wiley, New York, 1977).

${ }^{6} \mathrm{G}$. V. Frisk and $\mathrm{H}$. Uberall, "Creeping waves and lateral waves in acoustic scattering," J. Acoust. Soc. Am. 59, 46-54 (1976).

${ }^{7}$ M. F. Werby and L. H. Green, "A comparison of acoustical scattering from fluid-loaded elastic shells and sound soft objects," J. Acoust. Soc. Am. 76, 1227-1230 (1984).
${ }^{8}$ R. H. Hackman and G. S. Sammelmann, "Long-range scattering in a deep oceanic waveguide," J. Acoust. Soc. Am. 83, 1776-1792 (1988).

${ }^{9}$ R. H. Hackman and G. S. Sammelmann, "Acoustic scattering in a homogeneous waveguide," J. Acoust. Soc. Am 82, 324-336 (1987).

"W. A. Kuperman, M. F. Werby, and K. E. Gilbert, "Towed array response to ship noise: a near-field propagation problem," presented at NATO Conference on Underwater Acoustics Signal Processing, Luneberg, West Germany (1984).

"K. Marfurt, "Elastic wave equation migration-invertion," Ph.D. thesis, Columbia University, New York (1987).

${ }^{12}$ D. D. Kosloff and E. Baysal, "Forward modeling by a Fourier Method," Geophysics 47(10), 1402-1412 (1982).

${ }^{13} \mathrm{P}$. Carrion, "A layer-stripping technique for suppression of water-bottom multiple reflections," Geophys. Prospect. 34, 330-342 (1986).

${ }^{14} R$. A. Stephen, "Geoacoustic scattering from seafloor features in the ROSE area," J. Acoust. Soc. Am. 82, 238-256 (1987).

${ }^{15}$ R. M. Alford, K. R. Kelly, and D. M. Boore, "Accuracy of finite-difference modeling of the acoustic wave equation, Geophysics 39, 834-842 (1974). 